\title{
The tale of the three brothers - Shh, Wnt, and Fgf during development of the thalamus
}

\author{
Anja I. H. Hagemann and Steffen Scholpp* \\ Karlsruhe Institute of Technology, Institute of Toxicology and Genetics, Karlsruhe, Germany
}

Edited by:

Tomomi Shimogori, RIKEN Brain

Science Institute, Japan

\section{Reviewed by:}

David J. Price, The University of

Edinburgh, UK

James Y. H. Li, University of

Connecticut Health Center, USA

*Correspondence:

Steffen Scholpp, Karlsruhe Institute of Technology, Institute of Toxicology and Genetics, 76021 Karlsruhe,

Baden-Württemberg, Germany.

e-mail: steffen.scholpp@kit.edu
The thalamic complex is an essential part of the brain that requires a combination of specialized activities to attain its final complexity. In the following review we will describe the induction process of the mid-diencephalic organizer (MDO) where three different signaling pathways merge: Wnt, Shh, and Fgf. Here, we dissect the function of each signaling pathway in the thalamus in chronological order of their appearance. First we describe the Wnt mediated induction of the MDO and compartition of the caudal forebrain, then the Shh mediated determination of proneural gene expression before discussing recent progress in characterizing Fgf function during thalamus development. Then, we focus on transcription factors, which are regulated by these pathways and which play a pivotal role in neurogenesis in the thalamus. The three signaling pathways act together in a strictly regulated chronology to orchestrate the development of the entire thalamus.

Keywords: diencephalon, forebrain, thalamus, development, zebrafish, Shh, Wnt, Fgf

\section{INTRODUCTION}

In sixteenth century France, people liked listening to the fairy tale of the "Trois frères et le petite maison." In this story, in order to maintain and add to the splendor of their father's small house, three brothers had to compete for its inheritance by showing their skills as a barber, a blacksmith, and a swordsman. It was a difficult task since although all of them were excellent in one particular field; none of them could match their father's all-round skills and wisdom. So, it quickly became obvious that only upon combining their skills would their father's home attain its full splendor. Thus, the three brothers worked together happily in the small house until they grew old.

In analogy to "le petite maison," the thalamic complex is also a small but essential part of the brain that requires a combination of specialized activities to attain its final complexity. It is located at the top of the brainstem and can be subdivided into the anterior prethalamus and the posterior thalamus. The thalamic complex serves as the major relay station for sensory information in the brain, receiving nearly all sensory inputs and connecting them reciprocally with the overlying cortex. In contrast, the prethalamus sends hardly any axons to the cortex, but is defined by projections into the underlying thalamus. However, our knowledge about the development of the thalamic complex is still fragmented and here we will summarize the recent advances in the field.

During development, the brain becomes segmented along the anterior-posterior axis to ensure the formation of complex and functionally independent brain parts. The development of the thalamic complex serves as an example for the importance of this segmentation process. The thalamic complex in vertebrates is subdivided by a compartment boundary separating the prethalamus anteriorly from the thalamus posteriorly (Figures 1A-C). This transverse boundary between prethalamus and thalamus is called the Zona Limitans Intrathalamica (ZLI; Bergquist, 1932). According to the prosomeric model of Puelles and Rubinstein, the
ZLI is the border between prosomere 2 (P2) and prosomere 3 (P3; Puelles and Rubenstein, 1993, 2003).

Prominent morphological boundaries co-localize often with instructive cell populations - "local organizers" that are required to pattern adjacent brain regions. The isthmus with the midbrainhindbrain boundary organizer serves as a well-studied example for a compartment boundary with an organizer orchestrating the development of the adjacent tectum and cerebellum. It was suggested that the thalamus might similarly require instructive information from an organizer to orchestrate its patterning and maturation (Shimamura et al., 1995). A key feature of organizers is therefore their ability to influence nearby cell behavior in a non-cell autonomous manner through long-range signaling by secreted morphogens. Indeed, heterotopic transplantation of middiencephalic donor cells induces ectopic thalamic and prethalamic genes in surrounding host tissue (Garcia-Lopez et al., 2004; Vieira et al., 2005; Guinazu et al., 2007). At the ZLI we find a cell population, which expresses signaling members of the three protein families Wnt, Shh, and Fgf. These secreted proteins have been implicated in local organizing functions elsewhere in the brain. Also, cells within an organizer generally do not intermingle with those of adjacent regions. Such cell lineage restriction helps to stabilize the size and position of the organizer and ensures the production of a stable morphogen gradient from its source, which is imposed by the morphological characteristic of the ZLI. Cell lineage restriction has been shown to operate at the border of the ZLI (Zeltser et al., 2001; Garcia-Lopez et al., 2004). Thus, the cell population at the ZLI has all characteristics of a local organizer and is therefore termed mid-diencephalic organizer (MDO; also known as ZLI organizer).

In the following review we will describe the induction process of the MDO where three different signaling pathways merge: Wnt, Shh and Fgf (Scholpp and Lumsden, 2010) and we will dissect the function of each signaling pathway in the thalamus 
in chronological order of their appearance (Figure 2). We will therefore first describe the Wnt mediated induction of the MDO and compartition of the caudal forebrain, then the Shh mediated determination of proneural gene expression before discussing recent progress in characterizing Fgf function during thalamus development. We will then focus on transcription factors, which are regulated by these pathways and which play a pivotal role in neurogenesis in the thalamus. Like in the French tale, these three signaling factors act together in a strictly regulated chronology to orchestrate the development of the entire thalamus and none of them would be able to achieve such an elaborate structure on its own.

\section{INDUCTION AND POSITIONING OF THE MDO}

Prior to the formation of the anatomical ZLI boundary, the induction of the signaling center MDO in the diencephalon has been proposed to result from the interaction between the anterior (prechordal) and the posterior (epichordal) region of the neural plate. In ovo grafting and co-culture experiments in chick have shown that interaction between prechordal and epichordal neural tissues can induce Shh expression at their interface (Vieira et al., 2005; Guinazu et al., 2007). Molecular data obtained from experiments in different organisms may explain those co-culture results in a different way. Therefore, we have put a novel model forward incorporating findings from fish, chick, and mouse (Scholpp and Lumsden, 2010).

Firstly, by analyzing the function of transcription factors of the orthodenticle homeobox (Otx) family during regionalization of the neural plate in zebrafish, we found $O t x 1$ and Otx2 expression in the primordium of the MDO and thalamus (Scholpp et al., 2007). Indeed, down-regulation of Otx1/Otx2 immediately before MDO formation resulted in failure to induce the organizer. These findings are consistent with the analysis of Otx1-/-/Otx2 \pm double mutant mice, which also lack the MDO (Acampora et al., 1997). Since Otx genes are widely expressed in the forebrain and midbrain we asked the following questions. Firstly, what mechanism ensures the correct positioning of the MDO in the mid-diencephalon? Secondly, how is the tapering shape of the MDO established with a broad ventral domain and narrowing dorsal domain that coincides with shh expression?

Members of the Fez family of transcription factors are expressed in the prethalamic anlage from late gastrulation onward and abut the MDO territory (Hashimoto et al., 2000; MatsuoTakasaki et al., 2000; Staudt and Houart, 2007). Ectopic expression of FezF2 at the MDO in mice as well as its ubiquitous expression in fish leads to a block in organizer formation (Hirata et al., 2006;
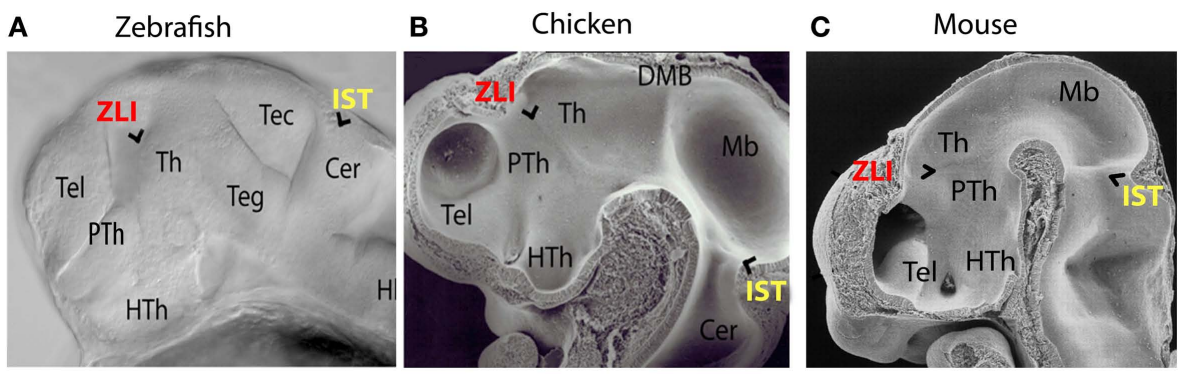

FIGURE 1 | Morphological comparison of embryonic brain regions in different vertebrate embryos. (A) Zebrafish $36 \mathrm{hpf}$. (B) Chicken $\mathrm{HH} 20$. (C) Mouse $11.5 \mathrm{dpf}$ (with courtesy of Salvador Martinez). In vertebrates the zona limitans intrathalamica (ZLI) forms as a morphological ridge between the prethalamus and the thalamus, whereas the isthmus rhombencephali (IST) marks the constriction between midbrain and hindbrain. Th, thalamus; PTh, prethalamus; HTh, Hypothalamus; Tel, Telencephalon; Teg, tegmentum; Tec, tectum; Cer, cerebellum; Mb, midbrain; DMB, diencephalic-mesencephalic boundary; Hi, hindbrain.
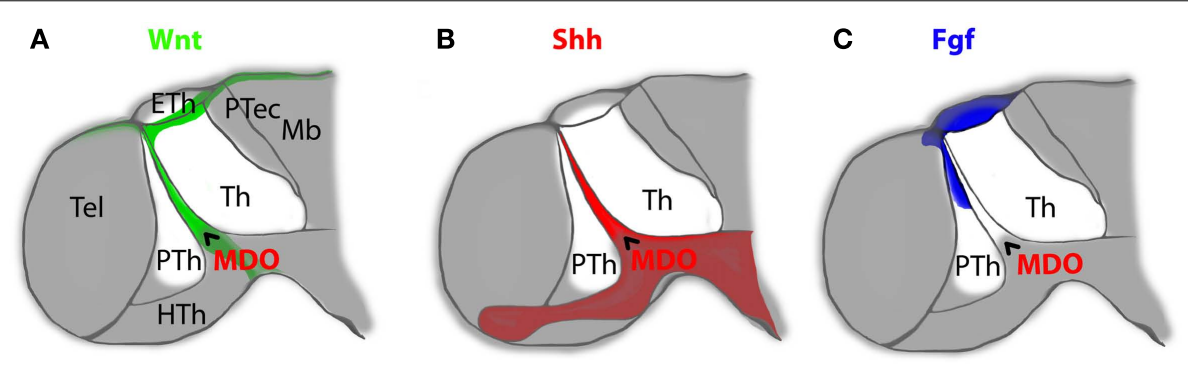

FIGURE 2 |The mid-diencephalic organizer (MDO) is the source for members of three morphogen signaling families in the ZLI of the embryonic vertebrate brain. Expression of Wnt signaling molecules [(A), green] overlaps with Shh producing cells of the MDO [(B), red], whereas expression of members of the Fgf family [(C), blue] is located in the prethalamus and overlaps partially with the Wnt signaling source in the dorsal roof plate. Taken together, these three families of signaling molecules encircle the developing thalamus from dorsal (Wnt and Fgf), ventral (Shh) and rostral (Wnt, Shh, and Fgf). Th, thalamus; PTh, prethalamus; HTh, Hypothalamus; Eth, epithalamus; Tel, Telencephalon; Ptec, pretectum; Mb, midbrain; MDO, mid-diencephalic organizer. 
Jeong et al., 2007). This suggests that Fez is required for setting the anterior border of the organizer by repressing the expression of organizer marker genes such as shh. Similarly, Irx1, a member of the transcription factor family of Iroquois genes, positions the posterior border of the MDO (Hirata et al., 2006; Scholpp et al., 2007). As shown in zebrafish and mouse, $\operatorname{Ir} x 1 b$ morphant embryos exhibit a posterior expansion of the MDO territory, as the transcriptional repressor is absent. Therefore, we conclude that the organizer of the thalamus is induced within an Otx-positive area that is subsequently refined to a small stripe between prethalamus and thalamus by the repressive function of Fez and Irx. Which mechanism subsequently ensures that the MDO primordium persists between Fez and Irx expression? During early somitogenesis in zebrafish, Wnt3 and Wnt3a are co-expressed in the MDO anlage and have a pivotal role in the induction of the MDO (Mattes et al., 2012). Wnt3/Wnt3a deficient embryos lack the MDO primordium and as a result the prethalamic territory abuts the thalamus. However, the size of the thalamus and prethalamus are unchanged arguing against a fate change of the MDO. Indeed, lack of canonical Wnt signaling leads to increased cell death of the MDO, suggesting Wnt3 and Wnt3a act as survival factors for organizer cells in fish (Mattes et al., 2012). Furthermore, in embryos deficient for Wnt3/Wnt3a/FezF2 or Wnt3/Wnt3a/Irx1b the MDO is restored. This suggests that the prethalamus as well as the thalamus are competent to form the organizer (Mattes et al., 2012). However, expression of Fez or Irx suppresses MDO development in these areas, hence shaping the MDO to a small strip of cells between prethalamus and thalamus. In the next paragraph, we will discuss the function of the MDO during thalamus development.

\section{Wnt/ $\beta$-CATENIN-SIGNALING, A NEW PLAYER IN THALAMIC DEVELOPMENT}

Wnt signaling is important to set up the initial anterio-posterior pattern of the neuraxis and it also has a more local function later during diencephalic regionalization (Wilson and Houart, 2004). Our knowledge of the latter is still fragmented while expression patterns of Wnt ligands, their receptors (Frizzled) and signaling mediators (Lef1, Tcf) in the mid-diencephalon do speak for a function in this location (Quinlan et al., 2009). Wnt3a and Wnt8b for example are expressed in the dorsal region of the thalamus and the MDO in mouse, chick, and fish from the precursor stage onward (Salinas and Nusse, 1992; Garcia-Lopez et al., 2004; Scholpp et al., 2007; Figure 2A). Furthermore, inhibition of canonical Wnt signaling by Dkk-1 in chick explants transforms the thalamus into prethalamus during the early regionalization phase (Braun et al., 2003). It has also been shown that the prethalamus marker Lhx5 can activate the expression of the extracellular Wnt inhibitors Sfrpla and Sfrp5 in zebrafish embryos (Peng and Westerfield, 2006). A significant demonstration of the importance of Wnt signaling in thalamus development is seen in zebrafish lacking Wnt3 and Wnt3a recently (Mattes et al., 2012). These embryos fail to develop an MDO. Wnt3/Wnt3a are required to maintain the MDO anlage in early somitogenesis by suppressing apoptosis of the organizer cells. Interestingly, this analysis has further shown that a narrow time window of $4 \mathrm{~h}$ of Wnt signaling is sufficient to trigger survival of the MDO cells. Similarly, mice lacking the essential Wnt co-receptor low-density lipoprotein receptor LRP6 fail to develop a normal MDO and thalamus (Zhou et al., 2004). These data suggest that canonical Wnt signaling is required for the induction of the MDO and for thalamus development, whereas the development of the prethalamus requires inhibition of canonical Wnt signaling. This hypothesis is supported by the observation that the expression of components of the Wnt signaling machinery as well as Wnt target gene expression are enriched in the thalamus, but absent in the prethalamus of all vertebrates analyzed so far from zebrafish to rhesus monkey (Jones and Rubenstein, 2004; Shimogori et al., 2004; Murray et al., 2007; Bluske et al., 2009; Quinlan et al., 2009; Peukert et al., 2011). The question remains, which aspects of canonical Wnt signaling are directly required for the development of the thalamus?

\section{CELL-CELL ADHESION IN THE CAUDAL THALAMUS}

Recently, Wnt signaling has been implicated in compartition of the caudal diencephalon. $\beta$-Catenin, the key effector of the Wnt pathway was initially discovered for its role in cell adhesion (Huber et al., 1996; Bienz, 2005). B-Catenin promotes adhesiveness by binding to the transmembrane, $\mathrm{Ca} 2+$-dependent homotypic adhesion molecule cadherin, and links cadherin to the intracellular actin cytoskeleton. Although several classes of molecules are involved in morphogenetic events, cadherins appear to be the major group of adhesion molecules mediating the formation of morphological and functional boundaries in the developing brain (Takeichi, 1977). Therefore it seemed reasonable to assume that Wnt signaling in combination with cadherin function regulates cell adhesion in the thalamus. Indeed, in the late developing diencephalon classical cadherins, such as Chd2, Chd6b, Chd7, mark areas of gray matter, which correspond to presumptive brain nuclei (Redies et al., 2000). However, these studies are not able to explain segmentation in the caudal forebrain. Recently, we could show that the function of other members of the cadherin superfamily, the non-clustered protocadherins, links compartition in the developing diencephalon to Wnt signaling.

During somitogenesis in zebrafish, a member of this group, pcdh10b (formerly known as OL-protocadherin) has been shown to modulate cell adhesion in the paraxial mesoderm and somite segmentation (Murakami et al., 2006). However, the expression of $p c d h 10 b$ also marks the alar part of $\mathrm{P} 2$, the thalamus, and we could show that canonical Wnt signaling regulates the expression of $p c d h 10 b$ in the developing thalamus of the zebrafish (Figure 3A). Stabilization of $B$-catenin leads to a broadening of the expression domain of $p c d h 10 b$ whereas inhibition of Wnt signaling blocks pcdh10b expression (Peukert et al., 2011). Hence, alteration of $p c d h 10 b$ expression in the thalamus leads to an intermingling of thalamic cells with the neighboring brain areas, predominantly with the pretectum in $\mathrm{P} 1$. Our data were supported by the fact that $p c d h 10 b$ knock-down or overexpression also lead to a similar intermingling of cells in developing somites (Murakami et al., 2006). Interestingly, $p c d h 10 b$ expression is strongest in thalamic progenitors, and non-detectable in mature post-mitotic neurons. Indeed, if progenitors are forced into differentiation by overexpression of Lhx2 we find a down-regulation of $p c d h 10 b$ expression (Peukert et al., 2011). Consistently, Lhx2/Lhx9 deficient embryos display a strong expression of $p c d h 10 b$ within the thalamus and in the adjacent brain areas as neuronal differentiation is blocked in 


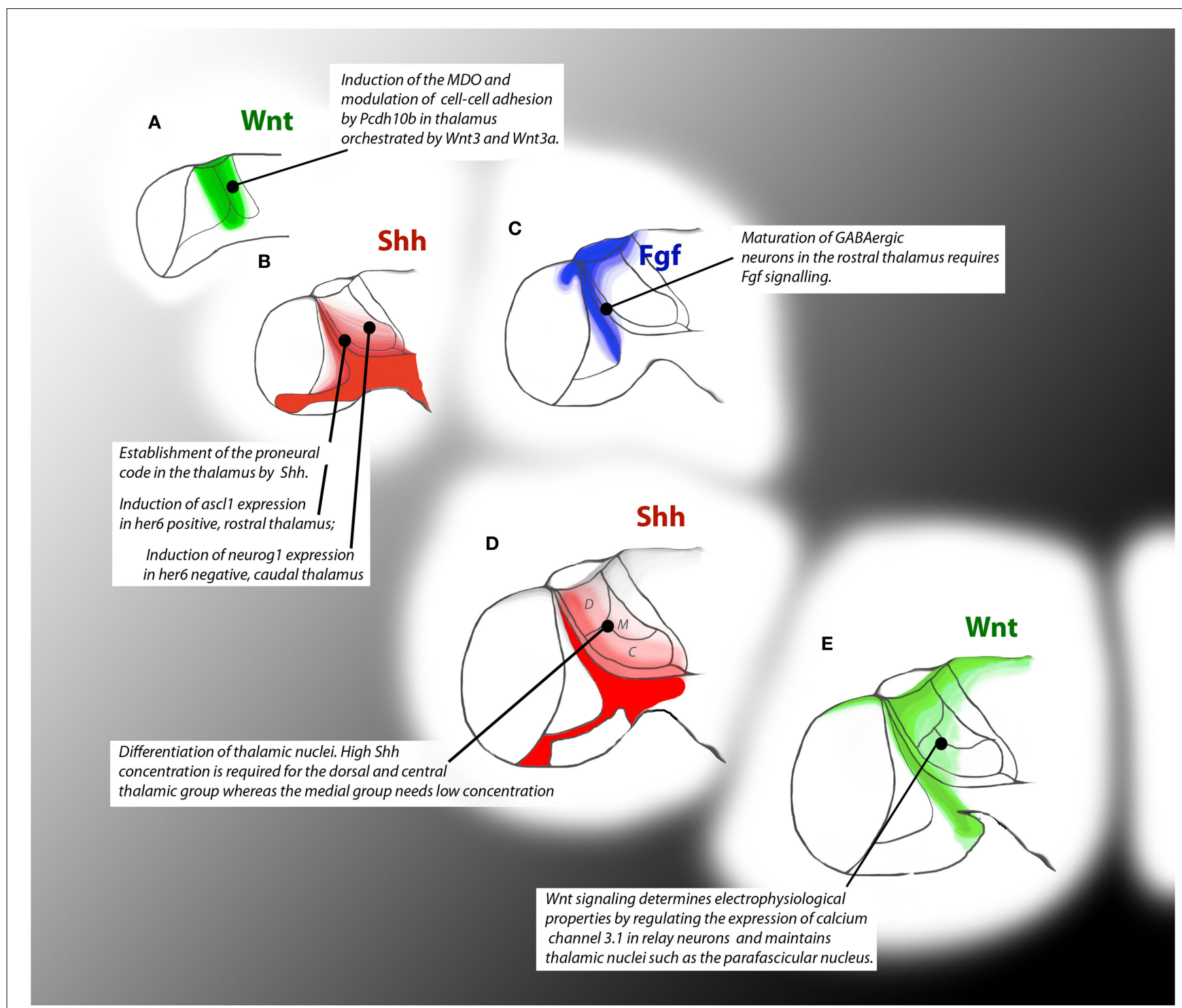

FIGURE 3 | Timeline of signaling events during thalamus formation in zebrafish embryos. Colored patterns show areas of influence of the depicted signaling pathways. At $15 \mathrm{hpf}$ Wnt signals from the caudal forebrain are required for the induction of the MDO and for compartition of the thalamus (A). At $24 \mathrm{hpf}$ Shh signaling is required for the onset of neurogenesis by induction of proneural genes (B) whereas Fgf signaling influences maturation of GABAergic neurons (C). At 36 hpf, graded Shh signal specifies the territory of the thalamic nuclei (D). At $48 \mathrm{hpf}, \mathrm{Wnt}$ signaling guides neurogenesis by influencing post-mitotic characteristics of thalamic neurons (E). these embryos. Thus, Wnt induced Pcdh10b helps to establish the thalamus as a compartment during forebrain development.

Similarly, in Gbx2 knock-out mice, thalamus cells start to intermingle with pretectum cells (Chen et al., 2009). Interestingly, these authors observe a non-cell autonomous function for this transcription factor and propose a restriction mechanism mediated by an unknown cell adhesion factor. Based on our recent data, we suggest that Gbx2 and Lhx2/Lhx9 are acting in a common pathway. Lack of Gbx2 in mice may lead therefore to a similar phenotype observed for the Lhx2/Lhx9 deficient morphant zebrafish embryos: an alteration of Wnt signaling in the caudal diencephalon which influences the expression of non-clustered Pcdhs and thus compartition.
In other vertebrate models, $P c d h 10$ expression has been reported only at later stages in development, in chicken $\mathrm{HH} 28$ and in mouse E15 (Hirano et al., 1999; Nakao et al., 2005), arguing against a comparable role in these model organisms. However, Pcdh10 together with Pcdh8, 12, 17, 18, and 19 belong to the group of structurally related non-clustered $\delta 2$ protocadherins and several members show expression during somitogenesis in mice (Kim et al., 2011). In contrast to the function of classical cadherins only a few Pcdhs show adhesive activity in vivo. This includes Xenopus Papc, which is regulated by non-canonical Wnt signaling (Schambony and Wedlich, 2007). Other Pcdhs appear to have more varied functions such as homophilic or heterophilic interactions. One possibility is that they may function as regulators of 
cell-cell adhesion by modulating cadherin adhesiveness. However, there is recent evidence that the intracellular domain of Pcdhs may contribute also to signaling (Kim et al., 2011). This role of Pcdh in signaling as well as the role of $B$-catenin in cell-cell adhesion within the thalamus should be explored further.

\section{REGIONALIZATION OF THE THALAMIC COMPLEX REOUIRES Shh SIGNALING}

Besides canonical Wnt ligands, the best-characterized signals the MDO releases into the thalamic complex are members of the Hedgehog family. Expression of hedgehog proteins has been found in the MDO of all vertebrate model organisms examined, including lamprey (Osorio et al., 2005), zebrafish (Barth and Wilson, 1995), frog (Ruiz and Altaba, 1998), chick (Puelles and Martinezde-la-Torre, 1987), and mouse (Shimamura et al., 1995). Furthermore, recent studies in chick (Kiecker and Lumsden, 2004; Vieira et al., 2005), fish (Scholpp et al., 2006), and in mice (Jeong et al., 2011) have shown that Shh released from the MDO regulates regionalization and patterning of the thalamus. Loss of Shh signaling results in the loss of genetic fate determinants and of thalamic identity in chick and fish (Hashimoto-Torii et al., 2003; Kiecker and Lumsden, 2004; Vieira et al., 2005; Scholpp et al., 2006). In addition, elevated Shh signaling expands thalamic identity in the forebrain: misexpression of Shh induces ectopic expression of thalamic markers such as Ascl1, Neurogenin, Abx2, Olig2, and Olig3 in the pretectum in chick and mouse (Echevarria et al., 2003; Vue et al., 2009). Thus, Shh is an important external cue that controls the expression of transcription factors within the thalamic complex.

Recent studies in mouse suggested that Shh is also required during regionalization of the thalamus. The thalamus can be subdivided into two different neuronal populations, the rostral thalamus (rTh) which is located close to the MDO and that contains mainly GABAergic inhibitory neurons and the caudal thalamus (cTh) which is located further away from the MDO and that represents the glutamatergic sensory thalamus (Vue et al., 2007). The activity of Shh as a morphogen has been linked directly to this differentiation process (Figure 3B). Reduction of Shh signaling from the MDO leads to a misspecification of GABAergic interneurons of the rTh and consequently to the loss of its derivative structures such as the ventral geniculate body and intergeniculate leaflet (Vue et al., 2009; Jeong et al., 2011). Thus, high concentrations of Shh are required for the formation of the rTh and low concentrations are required for the development of the cTh. These findings can be translated into a Gli transcription factor dependent activation of target genes: Activation of Glil leads to the induction of target genes of the rTh such as Sox14 and electroporation of Gli2 has been shown to activate cTh-markers such as Gbx2 in chick embryos (Hashimoto-Torii et al., 2003).

One pitfall of the thalamic morphogen gradient hypothesis is that concentration differences of only one morphogen within a field of cells fails to establish sharp borders between cell populations (Meinhardt, 2009). Which mechanism then is sharpening the border between the GABAergic rTh and the glutamatergic $\mathrm{cTh}$ ? The hairy/enhancer of split genes $\mathrm{E}(\mathrm{spl})$ may play an important role in neurogenesis and glia formation (Chapouton et al., 2011). We recently found that one of these bHLH proteins, the hairy-like factor 6 (Her6) in fish - the ortholog of Hes1 in mouse - regulates neuronal identities by determining expression of different proneural genes in the thalamus (Scholpp et al., 2009). Its expression is specifically maintained in the $\mathrm{rTh}$, whereas in the cTh it is down regulated prior to induction of proneural genes. Therefore, Her6 is a good candidate for the establishment of a sharp border between these thalamic territories. Indeed, Ascl1 is induced by Shh signaling from the MDO in the Her6 positive domains of the rTh, whereas in the Her6 negative cTh, Shh induces the expression of the proneural gene, neurogenin 1 . This expression code of proneural genes translates subsequently into the formation of GABAergic inhibitory neurons from the Ascl1 positive precursors in the rTh. The Neurogenin 1 positive cells will differentiate into glutamatergic relay neurons of the cTh. Interestingly, in the mouse retina it has been shown that Shh signaling stabilizes BHLH factors (Wall et al., 2009), suggesting a positive maintenance mechanism for the formation of Her6 positive, GABAergic cells close to the Shh source.

In the following chapter, we will discuss the important role of Fgf signaling in formation of the $r$ Th parallel to the Shh gradient and the BHLH Her6 dependent refinement mechanism.

\section{Fgf SIGNALING IN THALAMIC DEVELOPMENT}

Fgfs signaling is well known for its important roles during development of the central nervous system (Hebert, 2011) and, in addition to Shh and Wnt, has been implicated in the regulation of diencephalic development. Among the over 20 Fgf ligands, Fgf3, Fgf8 and the paralog genes Fgf15 and Fgf19 may play pivotal roles in this process. Early blockage of Fgf3 and Fgf8 mediated signaling for example leads to an alteration in prosencephalon patterning, including the absence of the prethalamic territory (Walshe and Mason, 2003). Later in development, Fgf15 and Fgf19 are expressed in the thalamus and prethalamus of mouse, chick, and fish (Ishibashi and McMahon, 2002; Miyake et al., 2005; Gimeno and Martinez, 2007). Their expression in the thalamus depends on long-range Shh signaling from the organizer. In fish, blockage of Fgf19 leads to a down-regulation of GABAergic inhibitory neurons in the prethalamus (Miyake et al., 2005). Likewise, electroporation of a truncated version of the Fgf-receptor-3 in the mouse thalamus leads to a lack of Ascll positive GABAergic neurons of the rTh (Figure 3C; Kataoka and Shimogori, 2008). Consistently, increased Fgf activity leads to a broadening of the rTh area and a caudal shift of sensory nuclei in the cTh (Kataoka and Shimogori, 2008). This suggests that Fgf signaling is required for the development of GABAergic neurons in the prethalamus and in the rTh, most likely downstream of Shh. In parallel to its function in inducing GABAergic neurons in the thalamic complex, Fgf signaling seems to have also an influence on the development of glutamatergic neurons in the cTh. Analysis of Fgf8 hypomorphic mice revealed a reduction of $G b \times 2$ expression in the thalamic neuroepithelium of the cTh (Martinez-Ferre and Martinez, 2009). The question remains about which Fgf ligand is required during the development of the thalamus? Fgf8 expression is found predominantly in the dorsal part of the diencephalon, in the most dorsal tip of the MDO and in the epithalamus, whereas Fgf15 and Fgf19 are expressed only in the ventral prethalamus. Therefore, Fgf8 supposedly exerts its function in the dorsal part whereas 
Fgf15 and Fgf19 influence rather the ventral area of the thalamus. However, the individual molecular mechanism of their local activity during diencephalic patterning still needs to be analyzed in detail. One may consider two experimental caveats for future analysis. Overexpression-based analyses may provide only insufficient answers as Fgf ligands tend to bind unspecifically to a number of Fgf receptors if the tissue is overloaded with the signaling molecule. Furthermore, timing of the experimental intervention as well as of the read-out is of great importance as Fgf signaling plays influential roles also during early phases of neural development. Another question for the future would be how Fgf signaling interacts with the other signaling pathways in order to pattern the nascent thalamus correctly? A first line of experiments in mice suggests that Fgf signaling may induce the expression of Wnt3a, whereas Shh expression in the MDO was not modified upon alteration in Fgf signaling (Kataoka and Shimogori, 2008; Martinez-Ferre and Martinez, 2009).

\section{LATE DEVELOPMENTAL PHASE - Lhx2/Lhx9 MEDIATED NEUROGENESIS}

As described above one consequence of the three external cues is the induction of certain transcription factors in the thalamus. For example, lack of Shh expression at the organizer leads to the absence of Neurog and Gbx2 in the thalamus in chick as well as zebrafish (Hashimoto-Torii et al., 2003; Kiecker and Lumsden, 2004; Vieira et al., 2005; Scholpp et al., 2006) and there is evidence that Fgf signaling may act upstream of Lhx2 in fish (Seth et al., 2006). Although there are many more transcription factors specifically expressed in the thalamus (Suzuki-Hirano et al., 2011), only a few have been functionally characterized, such as Gbx2, Neurog, Lhx2, Lhx9, and Lef1. Gbx2 knock-out mice lack the thalamus-specific post-mitotic neuronal markers Id4 and Lef1, and subsequently lack cortical innervations by thalamic axons (Miyashita-Lin et al., 1999). Furthermore, Gbx2 mediated differentiation is required for compartition of the caudal forebrain in mouse (Chen et al., 2009). Together with Shh, Gbx2 is important for the establishment of the thalamic nuclei in mouse (Figure 3D; Szabo et al., 2009; Vue et al., 2009). Although Neurog2 knock-out mice show a similarly severe failure in neuronal connectivity to the cortex, the expression of $L h \times 2, I d 2$, and $G b \times 2$ is unchanged in these animals, suggesting that in the absence of Neurog2 thalamic neurons are not re-specified at the molecular level (Seibt et al., 2003). Recently, the highly conserved apterous group proteins, Lhx2 and Lhx9, have been implicated in forebrain development. Lhx2 is required in mouse for the maintenance of cortical identity and to confine the cortical hem, allowing proper hippocampus formation in the adjacent pallium (Potter et al., 1997; Mangale et al., 2008). The Apterous transcripts are present in the nervous system of the cephalochordate Amphioxus, i.e., AmphiLhx2/9 (Takatori et al., 2008), and co-expression of $L h \times 2$ and $L h \times 9$ has been documented in the diencephalon of vertebrates, such as zebrafish (Ando et al., 2005), Xenopus (Bachy et al., 2001; Moreno et al., 2004), and mouse (Nakagawa and O'Leary, 2001). Thus, although recent studies of Lhx2-mutant mice showed no alteration during thalamic neuronal regionalization (Lakhina et al., 2007), co-expression of Lhx9 may compensate. Furthermore, although the function of Lhx9 has not been described, its expression pattern suggests a role during forebrain development (Nakagawa and O'Leary, 2001). We could indeed show now that in fish Lhx2 is functionally redundant to Lhx9 and that they regulate thalamic development. Single knock-down of Lhx2 or Lhx9 has no diencephalic phenotype but their simultaneous knock-down leads to stalling of thalamic neurogenesis at the late progenitor stage (Peukert et al., 2011). In the thalamus, $\operatorname{Lhx} 2 / \operatorname{Lh} x 9$ may regulate genes that are essential to complete neuronal development, such that $L h \times 2 / L h \times 9$ deficient neurons do not reach the terminal neuronal stage. Similar to the Gbx2-/- knock-out mouse (Miyashita-Lin et al., 1999), we find that the expression of $\operatorname{deltaA}$, neurog1, and $p c d h 10 b$ is increased in lhx2/lhx9 morphant zebrafish embryos (Peukert et al., 2011). During neuronal development in fish, neurog1 has been shown to activate delta genes directly by binding several E-box elements in the delta promoter region (Hans et al., 2004). This suggests that in lhx2/lhx9 morphant embryos, neuronal progenitor development is arrested at the level of deltaA/neurog 1 expression. Consequently, terminal thalamic neuronal markers such as Id2a and Lef1 are absent in Lhx2/Lhx9 morphant embryos. Thus, we propose that Lhx2/Lhx9 are essential determinants for neurons to reach the late stage of thalamic development. Interestingly, in the spinal cord Lim HD factors together with BHLH factors have been shown to be required for cell cycle exit in mouse (Lee and Pfaff, 2003). The Lim containing factor Isl- 1 and Lhx3 together with the BHLH factors Neurog2 and NeuroM act in a combinatorial manner to direct motor neuron differentiation. In the thalamus, we find a similar process: Lhx2/Lhx9 activate the expression of post-mitotic differentiation markers such as $i d 2 a$, lef1, and elavl3.

\section{Wnt SIGNALING AND NEUROGENESIS}

Evidence is accumulating that Wnt signaling plays an important role in the regulation of thalamic neurogenesis also at later stages. Analysis of a Wnt-reporter and also mosaic loss-of-function studies for $\beta$-catenin indicate strong activity of Wnt signaling in the ventricular zone of the neural tube (Freese et al., 2010). Similarly, pcdh10b, a novel Wnt target gene, is expressed in the ventricular zone of the thalamus (Peukert et al., 2011). Furthermore, differentiating neurons are characterized by the expression of members of the DNA-binding protein inhibitor genes (Id) as well as Lef1 (Jones and Rubenstein, 2004) and these markers are activated by Wnt signaling in the thalamus (Figure 3E; Peukert et al., 2011). However, the expression of these markers is most prominent in the subventricular zone as well as in the mantle zone. This suggests that Wnt signaling together with Lhx2/Lhx9 has a role in turning mitotically active neuronal precursors into mature post-mitotic neurons in the thalamus. Consistently, mice mutant for the Wnt co-receptor Lrp6 have a defective expression of thalamic neuronal markers such as Lef1 (Zhou et al., 2004). Interestingly, we observed an upregulation of Wnt activity in the $\operatorname{lh} \times 2 / \operatorname{lh} \times 9$ morphant embryos and consistently an upregulated expression of the Wnt target gene axin 2 as well as the novel target $p c d h 10 b$ in the ventricular zone of the thalamic neuroepithelium. However, further Wnt target genes, i.e., Lef1 are down regulated in the morphant embryos. This suggests that thalamic neuronal differentiation is coupled to a second competence phase for Wnt signaling in the mantle zone (Peukert et al., 2011). Also, the late and restricted onset of $\operatorname{lh} \times 2 / \operatorname{lh} \times 9$ expression in the thalamus and their requirement for $i d 2 a$ and $l e f 1$ 
Table 1 | Summary of functions of the three signaling pathways during thalamus development with related animal models in chronological order of publication.

\begin{tabular}{|c|c|c|c|}
\hline & Function & Model organism & Reference \\
\hline \multirow[t]{6}{*}{ Wnt } & Wnt signaling is important for patterning in the thalamic complex & Chicken & Braun et al. (2003) \\
\hline & Fate map of the Wnt8b positive MDO & Chicken & Garcia-Lopez et al. (2004) \\
\hline & Mutation in the Wnt co-receptor Lrp6 leads to loss of the thalamus & Mouse & Zhou et al. (2004) \\
\hline & Collection of expression patterns of members of the Wnt signaling pathway & Chicken & Quinlan et al. (2009) \\
\hline & $\begin{array}{l}\text { Compartition in the caudal forebrain requires the Wnt-dependent induction } \\
\text { of the adhesion modulator Pcdhl0b }\end{array}$ & Zebrafish & Peukert et al. (2011) \\
\hline & Wnt3 and Wnt3a are required for the induction and survival of the MDO & Zebrafish & Mattes et al. (2012) \\
\hline \multirow[t]{9}{*}{ Shh } & Shh marks the MDO at the prechordal/epichordal neural plate boundary & Mouse & Shimamura et al. (1995) \\
\hline & The Shh positive ZLI is a compartment in the caudal forebrain & Chicken & Zeltser et al. (2001) \\
\hline & Shh acts as a morphogen in thalamus regionalization & Chicken & Hashimoto-Torii et al. (2003) \\
\hline & Shh is the principal organizing signal of the MDO & Chicken & $\begin{array}{l}\text { Kiecker and Lumsden (2004), Vieira } \\
\text { et al. (2005) }\end{array}$ \\
\hline & Shh-a and Shh-b orchestrate thalamus regionalization & Zebrafish & Scholpp et al. (2006) \\
\hline & Shh influences the neural progenitor domains in the thalamus & Mouse & Vue et al. (2007) \\
\hline & $\begin{array}{l}\text { Shh is important for the differentiation of the medial and intralaminar } \\
\text { thalamic nuclei }\end{array}$ & Mouse & Szabo et al. (2009) \\
\hline & Her6 specifies the mode of Shh action in the rostral and caudal thalamus & Zebrafish & Scholpp et al. (2009) \\
\hline & $\begin{array}{l}\text { Shh signaling from the MDO and basal plate is important for the formation } \\
\text { of the rostral thalamus }\end{array}$ & Mouse & Jeong et al. (2011) \\
\hline \multirow[t]{2}{*}{ Fgf } & Maintenance of the rostral inhibitory thalamus is Fgf dependent & Mouse & Kataoka and Shimogori (2008) \\
\hline & $\begin{array}{l}\text { Fgf regulates Wnt3a expression in the MDO and influences thalamus } \\
\text { maturation }\end{array}$ & Chicken & Martinez-Ferre and Martinez (2009) \\
\hline
\end{tabular}

expression may explain the thalamic neuronal specificity of the Wnt target lef1. A consequence of Lef1 activity is the regulation of the expression of the Ca2+-channel Cav3.1 in mouse (Wisniewska et al., 2011). Therefore, we suggested that the second phase of Wnt signaling is required - via the activation of Lef1 - to reach the late stage of thalamic neuronal development and to determine the electrophysiological properties of thalamic neurons.

\section{SUMMARY}

Light has now been shed on the formation and function of the newly characterized MDO. Here, we summarized how the action of three different signaling pathways coordinates thalamic development through the MDO. Members of the three protein families, Wnt, Shh, and Fgf, are actively signaling in the mid-diencephalon and have different functions during development of the thalamic complex (Table 1). They regulate the compartition of the thalamus, drive neurogenesis, influence the neurotransmitter fate of

\section{REFERENCES}

Acampora, D., Avantaggiato, V., Tuorto, F., and Simeone, A. (1997). Genetic control of brain morphogenesis through Otx gene dosage requirement. Development 124, 3639-3650.

Ando, H., Kobayashi, M., Tsubokawa, T., Uyemura, K., Furuta, T., and Okamoto, H. (2005). Lhx2 mediates the activity of Six3 in zebrafish forebrain growth. Dev. Biol. 287, 456-468.
Bachy, I., Vernier, P., and Retaux, S. (2001). The LIM-homeodomain gene family in the developing Xenopus brain: conservation and divergences with the mouse related to the evolution of the forebrain. J. Neurosci. 21, 7620-7629.

Barth, K. A., and Wilson, S. W. (1995). Expression of zebrafish $\mathrm{nk} 2.2$ is influenced by sonic hedgehog/ vertebrate hedgehog-1 and demarcates a zone of neuronal differentiation in

thalamic neurons, and determine the electrophysiological characteristics of thalamic neurons. Considering the multifunctionality of these signaling pathways themselves and the expression of a cohort of further ligands of these pathways at the MDO, further research is needed into their possible function during thalamic development. In parallel, we still need to investigate how these signaling pathways influence each other. However, it has become clear that all three brothers together are needed to build the flourishing house of the thalamus, "le petite maison" of the brain.

\section{ACKNOWLEDGMENTS}

We would like to thank Gary Davidson (KIT) for critical reading of the manuscript, Salvador Martinez (University of Murcia, Spain) for providing the SEM picture of a mouse embryo at E11.5 and Daniela Peukert for her input regarding graphical layout. Anja I. H. Hagemann and Steffen Scholpp are supported by the DFG Emmy-Noether fellowship 847/2.

the embryonic forebrain. Development 121, 1755-1768.

Bergquist, H. (1932). Zur Morphologie Des Zwischenhirns Bei Niederen Wirbeltieren Stockholm. Acta Zool. 1, 74-221.

Bienz, M. (2005). Beta-Catenin: a pivot between cell adhesion and Wnt signalling. Curr. Biol. 15, R64-R67.

Bluske, K. K., Kawakami, Y., koyanoNakagawa, N., and Nakagawa, Y. (2009). Differential activity of
Wnt/beta-catenin signaling in the embryonic mouse thalamus. Dev. Dyn. 238, 3297-3309.

Braun, M. M., Etheridge, A., Bernard, A., Robertson, C. P., and Roelink, H. (2003). Wnt signaling is required at distinct stages of development for the induction of the posterior forebrain. Development 130, 5579-5587.

Chapouton, P., Webb, K. J., Stigloher, C., Alunni, A., Adolf, B., Hesl, B., Kremmer, E., and Bally-Cuif, L. (2011). 
Expression of hairy/enhancer of split genes in neural progenitors and neurogenesis domains of the adult zebrafish brain. J. Comp. Neurol.519, 1748-1769.

Chen, L., Guo, Q., and Li, J. Y. (2009). Transcription factor Gbx2 acts cell-nonautonomously to regulate the formation of lineagerestriction boundaries of the thalamus. Development 136, 1317-1326.

Echevarria, D., Vieira, C., Gimeno, L., and Martinez, S. (2003). Neuroepithelial secondary organizers and cell fate specification in the developing brain. Brain Res. Brain Res. Rev. 43, 179-191.

Freese, J. L., Pino, D., and Pleasure, S. J. (2010). Wnt signaling in development and disease. Neurobiol. Dis. 38, 148-153.

Garcia-Lopez, R., Vieira, C., Echevarria, D., and Martinez, S. (2004). Fate map of the diencephalon and the zona limitans at the 10 -somites stage in chick embryos. Dev. Biol. 268, 514-530.

Gimeno, L., and Martinez, S. (2007). Expression of chick Fgf19 and mouse Fgf15 orthologs is regulated in the developing brain by Fgf8 and Shh. Dev. Dyn. 236, 2285-2297.

Guinazu, M. F., Chambers, D., Lumsden, A., and Kiecker, C. (2007). Tissue interactions in the developing chick diencephalon. Neural Dev. 2, 25.

Hans, S., Scheer, N., Riedl, I. V., Weizsacker, E., Blader, P., and CamposOrtega, J. A. (2004). her3, a zebrafish member of the hairy-E(spl) family, is repressed by Notch signalling. Development 131, 2957-2969.

Hashimoto, H., Yabe, T., Hirata, T., Shimizu, T., Bae, Y., Yamanaka, Y., Hirano, T., and Hibi, M. (2000). Expression of the zinc finger gene fez-like in zebrafish forebrain. Mech. Dev. 97, 191-195.

Hashimoto-Torii, K., Motoyama, J., Hui, C. C., Kuroiwa, A., Nakafuku, M., and Shimamura, K. (2003). Differential activities of Sonic hedgehog mediated by Gli transcription factors define distinct neuronal subtypes in the dorsal thalamus. Mech. Dev. 120, 1097-1111.

Hebert, J. M. (2011). FGFs: Neurodevelopment's Jack-of-all-Trades - how do they do it? Front. Neurosci. 5:133. doi:10.3389/fnins.2011.00133

Hirano, S., Yan, Q., and Suzuki, S. T. (1999). Expression of a novel protocadherin, OL-protocadherin, in a subset of functional systems of the developing mouse brain. J. Neurosci. 19, 995-1005.

Hirata, T., Nakazawa, M., Muraoka, O., Nakayama, R., Suda, Y., and Hibi,
M. (2006). Zinc-finger genes Fez and Fez-like function in the establishment of diencephalon subdivisions. Development 133, 3993-4004.

Huber, O., Bierkamp, C., and Kemler, R. (1996). Cadherins and catenins in development. Curr. Opin. Cell Biol. 8, 685-691.

Ishibashi, M., and McMahon, A. P. (2002). A sonic hedgehogdependent signaling relay regulates growth of diencephalic and mesencephalic primordia in the early mouse embryo. Development 129, 4807-4819.

Jeong, J. Y., Einhorn, Z., Mathur, P., Chen, L., Lee, S., Kawakami, K., and Guo, S. (2007). Patterning the zebrafish diencephalon by the conserved zinc-finger protein Fezl. Development 134, 127-136.

Jeong, Y., Dolson, D. K., Waclaw, R. R., Matise, M. P., Sussel, L., Campbell, K., Kaestner, K. H., and Epstein, D. J. (2011). Spatial and temporal requirements for sonic hedgehog in the regulation of thalamic interneuron identity. Development 138, 531-541.

Jones, E. G., and Rubenstein, J. L. (2004). Expression of regulatory genes during differentiation of thalamic nuclei in mouse and monkey. J. Comp. Neurol. 477, 55-80.

Kataoka, A., and Shimogori, T. (2008). Fgf8 controls regional identity in the developing thalamus. Development 135, 2873-2881.

Kiecker, C., and Lumsden, A. (2004). Hedgehog signaling from the ZLI regulates diencephalic regional identity. Nat. Neurosci. 7, 1242-1249.

Kim, S. Y., Yasuda, S., Tanaka, H., Yamagata, K., and Kim, H. (2011). Nonclustered protocadherin. Cell Adh. Migr. 5, 97-105.

Lakhina, V., Falnikar, A., Bhatnagar, L., and Tole, S. (2007). Early thalamocortical tract guidance and topographic sorting of thalamic projections requires LIM-homeodomain gene Lhx2. Dev. Biol. 306, 703-713.

Lee, S. K., and Pfaff, S. L. (2003). Synchronization of neurogenesis and motor neuron specification by direct coupling of bHLH and homeodomain transcription factors. $\mathrm{Neu}$ ron 38, 731-745.

Mangale, V. S., Hirokawa, K. E., Satyaki, P. R., Gokulchandran, N., Chikbire, S., Subramanian, L., Shetty, A. S., Martynoga, B., Paul, J., Mai, M. V., Li, Y., Flanagan, L. A., Tole, S., and Monuki, E. S. (2008). Lhx2 selector activity specifies cortical identity and suppresses hippocampal organizer fate. Science 319, 304-309.
Martinez-Ferre, A., and Martinez, S. (2009). The development of the thalamic motor learning area is regulated by Fgf8 expression. J. Neurosci. 29, 13389-13400.

Matsuo-Takasaki, M., Lim, J. H., Beanan, M. J., Sato, S. M., and Sargent, T. D. (2000). Cloning and expression of a novel zinc finger gene, Fez, transcribed in the forebrain of Xenopus and mouse embryos. Mech. Dev. 93, 201-204.

Mattes, B., Weber, S., Chen, Q., Davidson, G., Houart, C., and Scholpp S. (2012). Wnt3 and Wnt3a are required for induction of the middiencephalic organizer in the caudal forebrain. Neural Dev. 7, 12

Meinhardt, H. (2009). Models for the generation and interpretation of gradients. Cold Spring Harb. Perspect. Biol. 4, 1-14.

Miyake, A., Nakayama, Y., Konishi, M., and Itoh, N. (2005). Fgf19 regulated by $\mathrm{Hh}$ signaling is required for zebrafish forebrain development. Dev. Biol. 288, 259-275.

Miyashita-Lin, E. M., Hevner, R., Wassarman, K. M., Martinez, S., and Rubenstein, J. L. (1999). Early neocortical regionalization in the absence of thalamic innervation. Science 285, 906-909.

Moreno, N., Bachy, I., Retaux, S. and Gonzalez, A. (2004). LIMhomeodomain genes as developmental and adult genetic markers of Xenopus forebrain functional subdivisions. J. Comp. Neurol. 472, 52-72.

Murakami, T., Hijikata, T., Matsukawa, M., Ishikawa, H., and Yorifuji, H. (2006). Zebrafish protocadherin 10 is involved in paraxial mesoderm development and somitogenesis. Dev. Dyn. 235, 506-514.

Murray, K. D., Choudary, P. V., and Jones, E. G. (2007). Nucleus- and cell-specific gene expression in monkey thalamus. Proc. Natl. Acad. Sci. U.S.A. 104, 1989-1994.

Nakagawa, Y., and O'Leary, D. D. (2001). Combinatorial expression patterns of LIM-homeodomain and other regulatory genes parcellate developing thalamus. J. Neurosci. 21, 2711-2725.

Nakao, S., Uemura, M., Aoki, E. Suzuki, S. T., Takeichi, M., and Hirano, S. (2005). Distribution of OL-protocadherin in axon fibers in the developing chick nervous system. Brain Res. Mol. Brain Res. 134, 294-308.

Osorio, J., Mazan, S., and Retaux, S. (2005). Organisation of the lamprey (Lampetra fluviatilis) embryonic brain: insights from
LIM-homeodomain, Pax and hedgehog genes. Dev. Biol. 288, 100-112.

Peng, G., and Westerfield, M. (2006). Lhx 5 promotes forebrain development and activates transcription of secreted Wnt antagonists. Development 133, 3191-3200.

Peukert, D., Weber, S., Lumsden, A., and Scholpp, S. (2011). Lhx2 and lhx 9 determine neuronal differentiation and compartition in the caudal forebrain by regulating wnt signaling. PLoS Biol. 9, e1001218. doi:10.1371/journal.pbio.1001218

Potter, P. E., Thorne, B., and Gaughan, C. (1997). Modulation of hippocampal norepinephrine release by cholinergic agonists is altered by AF64A lesion. Brain Res. Bull. 42, 153-160.

Puelles, L., and Martinez-de-la-Torre, M. (1987). Autoradiographic and Golgi study on the early development of n. Isthmi principalis and adjacent grisea in the chick embryo: a tridimensional viewpoint. Anat. Embryol. (Berl.) 176, 19-34.

Puelles, L., and Rubenstein, J. L. (1993). Expression patterns of homeobox and other putative regulatory genes in the embryonic mouse forebrain suggest a neuromeric organization. Trends Neurosci. 16, 472-479.

Puelles, L., and Rubenstein, J. L. (2003). Forebrain gene expression domains and the evolving prosomeric model. Trends Neurosci. 26, 469-476.

Quinlan, R., Graf, M., Mason, I., Lumsden, A., and Kiecker, C. (2009). Complex and dynamic patterns of Wnt pathway gene expression in the developing chick forebrain. Neural Dev. 4, 35.

Redies, C., Ast, M., Nakagawa, S., Takeichi, M., Martinez-de-laTorre, M., and Puelles, L. (2000). Morphologic fate of diencephalic prosomeres and their subdivisions revealed by mapping cadherin expression. J. Comp. Neurol. 421, 481-514.

Ruiz, I., and Altaba, A. (1998). Combinatorial Gli gene function in floor plate and neuronal inductions by Sonic hedgehog. Development 125, 2203-2212.

Salinas, P. C., and Nusse, R. (1992). Regional expression of the Wnt3 gene in the developing mouse forebrain in relationship to diencephalic neuromeres. Mech. Dev. 39, 151-160.

Schambony, A., and Wedlich, D. (2007). Wnt-5A/Ror2 regulate expression of XPAPC through an alternative noncanonical signaling pathway. Dev. Cell 12, 779-792. 
Scholpp, S., Delogu, A., Gilthorpe, J., Peukert, D., Schindler, S., and Lumsden, A. (2009). Her6 regulates the neurogenetic gradient and neuronal identity in the thalamus. Proc. Natl. Acad. Sci. U.S.A. 106, 19895-19900.

Scholpp, S., Foucher, I., Staudt, N., Peukert, D., Lumsden, A., and Houart, C. (2007). Otx1l, Otx2 and Irxlb establish and position the ZLI in the diencephalon. Development 134, 3167-3176.

Scholpp, S., and Lumsden, A. (2010). Building a bridal chamber: development of the thalamus. Trends Neurosci. 33, 373-380.

Scholpp, S., Wolf, O., Brand, M., and Lumsden, A. (2006). Hedgehog signalling from the zona limitans intrathalamica orchestrates patterning of the zebrafish diencephalon. Development 133, 855-864.

Seibt, J., Schuurmans, C., Gradwhol, G., Dehay, C., Vanderhaeghen, P., Guillemot, F., and Polleux, F. (2003). Neurogenin2 specifies the connectivity of thalamic neurons by controlling axon responsiveness to intermediate target cues. Neuron 39, 439-452.

Seth, A., Culverwell, J., Walkowicz, M., Toro, S., Rick, J. M., Neuhauss, S. C., Varga, Z. M., and Karlstrom, R. O. (2006). Belladonna/(Ihx2) is required for neural patterning and midline axon guidance in the zebrafish forebrain. Development 133, 725-735.

Shimamura, K., Hartigan, D. J., Martinez, S., Puelles, L., and Rubenstein, J. L. (1995). Longitudinal organization of the anterior neural plate and neural tube. Development 121, 3923-3933.

Shimogori, T., Vansant, J., Paik, E., and Grove, E. A. (2004). Members of the Wnt, Fz, and Frp gene families expressed in postnatal mouse cerebral cortex. J. Comp. Neurol. 473, 496-510.

Staudt, N., and Houart, C. (2007). The prethalamus is established during gastrulation and influences diencephalic regionalization. PLoS Biol. 5, e69. doi:10.1371/journal.pbio.0050069

Suzuki-Hirano, A., Ogawa, M., Kataoka, A., Yoshida, A. C., Itoh, D., Ueno, M., Blackshaw, S., and Shimogori, T. (2011). Dynamic spatiotemporal gene expression in embryonic mouse thalamus. J. Comp. Neurol. 519, 528-543.

Szabo, N. E., Zhao, T., Zhou, X., and Alvarez-Bolado, G. (2009). The role of Sonic hedgehog of neural origin in thalamic differentiation in the mouse. J. Neurosci. 29, 2453-2466.

Takatori, N., Butts, T., Candiani, S., Pestarino, M., Ferrier, D. E., Saiga, H., and Holland, P. W. (2008). Comprehensive survey and classification of homeobox genes in the genome of amphioxus, Branchiostoma floridae. Dev. Genes Evol. 218, 579-590.

Takeichi, M. (1977). Functional correlation between cell adhesive properties and some cell surface proteins. J. Cell Biol. 75, 464-474.

Vieira, C., Garda, A. L., Shimamura, K., and Martinez, S. (2005). Thalamic development induced by Shh in the chick embryo. Dev. Biol. 284, 351-363.

Vue, T. Y., Aaker, J., Taniguchi, A. Kazemzadeh, C., Skidmore, J. M. Martin, D. M., Martin, J. F., Treier, M., and Nakagawa, Y. (2007). Characterization of progenitor domains in the developing mouse thalamus. J. Comp. Neurol. 505, 73-91.

Vue, T. Y., Bluske, K., Alishahi, A., Yang, L. L., Koyano-Nakagawa, N., Novitch, B., and Nakagawa, Y. (2009). Sonic hedgehog signaling controls thalamic progenitor identity and nuclei specification in mice. J. Neurosci. 29, 4484-4497.

Wall, D. S., Mears, A. J., Mcneill, B., Mazerolle, C., Thurig, S., Wang, Y., Kageyama, R., and Wallace, V. A. (2009). Progenitor cell proliferation in the retina is dependent on Notchindependent Sonic hedgehog/Hes1 activity. J. Cell Biol. 184, 101-112.

Walshe, J., and Mason, I. (2003). Unique and combinatorial functions of Fgf3 and Fgf8 during zebrafish forebrain development. Development 130, 4337-4349.

Wilson, S. W., and Houart, C. (2004). Early steps in the development of the forebrain. Dev. Cell 6, 167-181.

Wisniewska, M. B., Misztal, K., Michowski, W., Szczot, M., Purta, E., Lesniak, W., Klejman, M. E., Dabrowski, M., Filipkowski, R. K., Nagalski, A., Mozrzymas, J. W., and Kuznicki, J. (2011). LEF1/beta-catenin complex regulates transcription of the Cav3.1 calcium channel gene (Cacnalg) in thalamic neurons of the adult brain. J. Neurosci. 30, 4957-4969.

Zeltser, L. M., Larsen, C. W., and Lumsden, A. (2001). A new developmental compartment in the forebrain regulated by Lunatic fringe. Nat. Neurosci. 4, 683-684.

Zhou, C. J., Pinson, K. I., and Pleasure, S. J. (2004). Severe defects in dorsal thalamic development in lowdensity lipoprotein receptor-related protein-6 mutants. J. Neurosci. 24 7632-7639.

Conflict of Interest Statement: The authors declare that the research was conducted in the absence of any commercial or financial relationships that could be construed as a potential conflict of interest.

Received: 10 February 2012; accepted: 04 May 2012; published online: 28 May 2012.

Citation: Hagemann AIH and Scholpp S (2012) The tale of the three brothers Shh, Wnt, and Fgf during development of the thalamus. Front. Neurosci. 6:76. doi: 10.3389/fnins.2012.00076

This article was submitted to Frontiers in Neurogenesis, a specialty of Frontiers in Neuroscience.

Copyright (c) 2012 Hagemann and Scholpp. This is an open-access article distributed under the terms of the Creative Commons Attribution Non Commercial License, which permits noncommercial use, distribution, and reproduction in other forums, provided the original authors and source are credited. 